\title{
Near specular scatter analysis method with a new goniophotometer
}

\author{
Stephanie Meyen* ${ }^{\mathrm{a}}$, Florian Sutter ${ }^{\mathrm{a}}$, Peter Heller $^{\mathrm{a}}$ \\ aDLR German Aerospace Center, Institute of Solar Research at Plataforma Solar de Almería (PSA), \\ Carretera Senés km 5, 04200 Tabernas, Spain
}

\begin{abstract}
The challenge of improving component quality and reducing cost has focused the attention of the solar thermal power industry on reliable component characterization methods. Since the reflector plays a key role in the energy conversion chain, the analysis of its reflectance properties has become a lively discussed issue in recent years. State of the art measurement instruments for specular reflectance do not give satisfying results, because they do not resolve sufficiently the near specular scatter of possible low cost mirror material candidates. The measurement of the BRDF offers a better solution than the traditional approach of placing a detector in the specular reflected beam path. However, due to the requirement of high angular resolution in the range of $1 \mathrm{mrad}\left(0.057^{\circ}\right)$ or better and the challenge of measuring high dynamic differences between the specular peak and the scatter signal, typical commercial scanning goniophotometers capable of this are rare. These instruments also face the disadvantages of impractically long acquisition times and, to reach the high angular resolution, occupy a large space (several meters side length). We have taken on the appealing idea of a parallel imaging goniophotometer and designed a prototype based on this principle. A mirrored ellipsoid is used to redirect the reflected light coming from a sample towards a camera with a fisheye lens. This way the complete light distribution is captured simultaneously. A key feature allows the distinction of the high intensity specular peak and the low intensity scatter. In this article we explain the prototype design and demonstrate its functionality based on comparison measurements done with a commercial scanning goniophotometer. We identify limitations related in part to the concept and in part to the specific prototype and suggest improvements. Finally we conclude that the concept is well suitable for the analysis of near specular scatter of mirror materials, although less adequate for the analysis of rough surfaces that require a full $180^{\circ}$ view angle. Results obtained with this instrument are useful to evaluate the performance of a reflector material for a specific concentrating solar collector design and also serve in other applications that require near specular scatter analysis like degradation and soiling research.
\end{abstract}

Keywords: reflectance measurement, near specular scatter, BRDF analysis, goniophotometer, metrological instrumentation

\section{INTRODUCTION}

The reflector is a key component in the energy conversion process of concentrating solar power (CSP) technology (also known as solar thermal power technology). In all common concentrator designs, a parabolic shaped mirror which follows the movement of the sun focusses the direct sunlight towards a receiver. The receiver consists of an absorber material which is heated up by the sun's energy and transfers the heat to a transport medium, which then fuels a common steam turbine power generation process [1]. Any deviation in the redirection of the sunlight towards the receiver reduces the efficiency of the CSP-System, which means a loss of profit. While other sources of beam deviation (i.e. shape errors, tracking errors, misplacement of receiver) have already been well understood and evaluated, new and innovative reflector materials that sometimes display near specular scattering still need intensive research on a detailed characterization of their specular reflectance properties.

Typical solar reflectors in the past were built out of second surface silvered glass mirrors of thicknesses between 3-5mm, manufactured by few established providers. Scattering did not play a role on these mirrors and little attention was paid to the detailed characterization of their specular reflectance properties. Actual efforts to reduce installation and maintenance cost for large scale solar thermal power plants require innovative collector designs constructed out of innovative materials. This also concerns the mirrors. New providers enter the market with innovative reflector materials, i.e. enhanced aluminum panel sheets or silvered polymeric films. Here, surface structures can lead to a widening of the specular reflected beam and to near specular, sometimes anisotropic scattering of the reflected radiation. A certain amount of narrow scattering does not influence the efficiency, because the geometry of the receiver itself accepts 
deviations up to a certain offset angle. This however depends on the individual system design and includes also other deviation sources as mentioned above. Therefore knowledge of the specular reflectance and scatter characteristic of the material in question is necessary. Since the reflectance characteristic of a material depends on the wavelength, $\lambda$, the incident angle, $\theta_{i}=\theta_{r}$, of the illumination and on the acceptance angle, $\varphi$ (offset half angle from the ideal specular direction), all these parameters need to be addressed in a measurement (Figure 1). Within the international cooperative network SolarPACES, experts from the main research organizations and industry developed a reflectance measurement guideline [2], which defines the relevant parameters for solar reflectors. These are the hemispherical reflectance, $\rho_{h}(\lambda, \theta, h)$, and the specular reflectance, $\rho_{s}(\lambda, \theta, \varphi)$, to be measured at least at near normal incidence (ideally as a function of $\theta$ ), in the solar wavelength range of $\lambda=300-2500 \mathrm{~nm}$ and as a function of $\varphi$ (or at least at discrete $\varphi$ in the range of $1-$ $25 \mathrm{mrad})$.

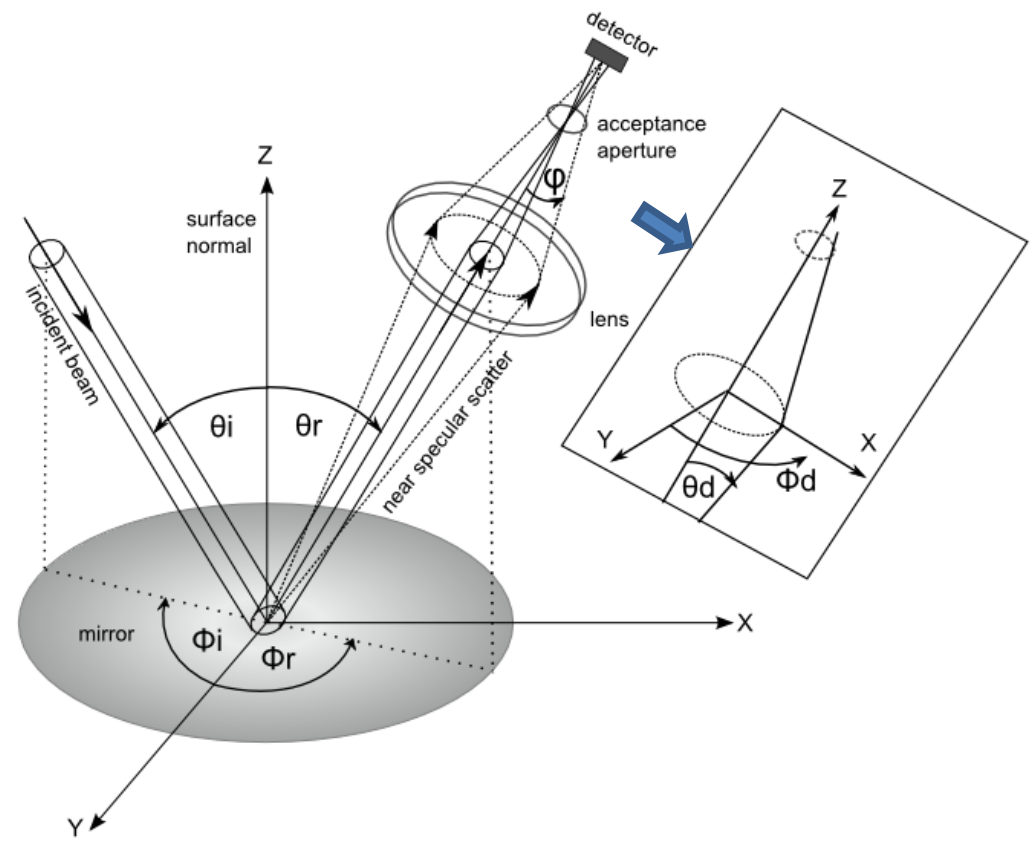

Figure 1: Schematic of specular reflection and common measurement method. A parallel incident beam intercepts the mirror sample at an incidence angle, $\theta_{i}$, is reflected in the reflection angle, $\theta_{r}$, and is then focused by a lens system onto a detector or into an acceptance aperture stop. Sometimes the incidence beam is focused directly into the aperture stop with the mirror sample placed in the beam path behind the lens system. In all cases the acceptance aperture of the detector or the aperture stop limit the offset angle of the perfectly specular beam, in which scattered light is still recognized by the detector. This maximum offset angle is called acceptance angle, $\varphi$. When centering the coordinate system around the specular peak, then the direction of scattered light beams can be described by the two angles $\left\{\theta_{d}, \Phi_{d}\right\}$.

Off the shelf instruments for measuring $\rho_{s}(\lambda, \theta, \varphi)$ most commonly measure the accumulated flux that intercepts a detector placed in the specular beam path [3]. The sensitive area is limited by the detector dimensions or an aperture stop (acceptance aperture) as indicated in Figure 1. Thus, the measured specular reflectance is related to a singular acceptance angle, $\varphi$. Some instruments that specifically account for $\varphi$, typically by employing variable apertures, lack a complete wavelength spectrum and variable incidence angles. The obtained result is often not sufficient for a valid evaluation of a reflector material for its application in a CSP system or for reliable comparisons of results that have been obtained with different types of instruments. This gave rise to recent intensive research on methods for a more detailed characterization of mirror reflectance properties [4][5], where the OPAC (optical and durability characterization) laboratory has taken on a leading role. OPAC is a collaboration project run by the German organization DLR and the Spanish organization CIEMAT at the Plataforma Solar de Almería in Spain.

In this context, the here presented measurement instrument prototype was developed at the OPAC laboratory, to provide an alternative to existing specular reflectance measurement instruments, that is adapted to the needs of CSP reflector analysis. The instrument follows a goniophotometric approach for analyzing the BRDF of a mirror sample. This has the advantage that the specular reflectance can be calculated for any desired acceptance angle and thus as function of the 
same. This is based on the assumption that the total integrated BRDF equates the hemispherical reflectance. The goniometric approach also eases the incorporation of variable incidence angles in the setup.

This document presents the newly developed prototype MIRA (Mirror Reflectacence Function Analyzer), which is a form of a so called parallel catadiopic mapping goniophotometer [6][7]. It employs an ellipsoidal mirror to redirect the scattered light coming from a sample towards a camera and is able to capture the BRDF simultaneously instead of scanning the hemisphere. This concept promises a relevant reduction of measurement time compared with traditional scanning goniophotometers, as well as a high signal throughput, a compact and robust design that still provides high angular resolution and does not rely on precision moving mechanics.

Literature about other instrument designs using this principle exists but we did not find information on any prototype that has been built and is functional in the visible with similarly high resolution. Our results show that the principle and this prototype setup works for the reflectance characterization of mirror materials that display near specular scatter. The reflectance can be obtained as a function of acceptance angle at variable incidence angles up to $\theta_{i} \sim 45^{\circ}$ and variable wavelengths in the visible range. Still, some limitations are identified, which in part are related to the physical properties of the components and in part to the specific prototype setup. The latter can be dealt with by following our suggestions for an improved version of the instrument prototype. We can conclude that the MIRA prototype provides a useful tool to characterize solar reflector or other specular materials and also evaluate the effect of degradation on scattering. This is possible within the boundary conditions discussed in this document.

The next sections explain the theory and design consideration of the setup, compare results obtained with MIRA on exemplary reflector samples with measurements that have been performed on the same samples with a scanning goniophotometer at pab Advanced Technologies Inc. and discuss limitations inflicted by this concept together with suggestions for improvement.

\section{THE INSTRUMENT PROTOTYPE}

From experience we have learned that typical alternative solar reflector materials display a narrow specular peak with a diameter of a few milliradiants [4][8] but can also produce scattering at angles $\varphi>>30$ mrad from peak. To find out more about the near specular scattering of these samples, an angular resolution around $1 \mathrm{mrad}\left(0.06^{\circ}\right)$ or better is required. The BRDF measurement with a scanning goniophotometer is time consuming and even if the direction of incidence radiation is set to a fixed value, still requires a moving arm with 2 dimensions of freedom. The longer the arm, the easier is a high angular resolution achieved. At PAB Advanced Technologies Inc. we found one of the few scanning goniophotometers that is able to perform BRDF measurements with a resolution even better than required in the dimension of $0.1 \mathrm{mrad}$. However, this instrument occupies a hall with 3.5 meter ceiling height and a minimum room length of 5 meter [9][10]. This space is not available in every laboratory. The concept of a parallel catadiopic imaging goniophotometer can feature a more compact design and still provide a high angular resolution together with a reduced measurement time due to the simultaneous capturing of the BRDF in one or a few images.

The following sections explain the theory behind the development of the new imaging goniophotometer and give a detailed overview on the necessary design considerations. Although the instrument features a movable light source that can be set to varying incidence angles and a filter system to create monochromatic illumination at variable wavelength bands in the range from $\lambda=500-700 \mathrm{~nm}$, these features are not specifically addressed in this document. The focus lies on the on the measurement principle itself.

\subsection{Theory}

The angular distribution of the reflected light flux coming from a sample is described by the BRDF (bidirectional reflectance distribution function). It can equally be measured with a traditional scanning goniophotometer or with a parallel imaging goniophotometer, where the angular distribution is captured simultaneously with a 2-dimensional detector array. The measurement of the BRDF is usually the necessary for the evaluation of rough surfaces and diffuse reflecting materials, where it is required to cover the whole hemisphere above the sample. Near angular scatter analysis of specular reflecting samples in turn require a high angular resolution in the area near the specular direction but the $\mathrm{BRDF}$ is zero in most of the rest of the hemisphere. The BRDF is commonly defined by equation (1): 


$$
\operatorname{BRDF}\left(\theta_{i}, \Phi_{i}, \theta_{r}, \Phi_{r}\right)=\frac{d L_{r}\left(\theta_{r}, \Phi_{r}\right)}{d E_{i}\left(\theta_{i}, \Phi_{i}\right)}
$$

Where $d L_{r}$ is the radiance per solid angle and per unit projected area, leaving the surface in the direction defined by the elevation angle, $\theta_{r}$, to the surface normal and the azimuth angle, $\phi_{r}$, while the surface is illuminated with the irradiance $d E_{i}$ per unit surface area coming from the direction $\left(\theta_{i}, \phi_{i}\right)$. A complete BRDF is therefore a four dimensional function. In many cases it is sufficient to reduce this function to two dimensions that only depend on $\theta_{r}$ and $\phi_{r}$ and can be measured with irradiance coming from a fixed position in space. Equation (1) can be rewritten by substituting radiance and irradiance by their definitions to

$$
\operatorname{BRDF}\left(\theta_{r}, \Phi_{r}\right)=\frac{d P_{r}\left(\theta_{r}, \Phi_{r}\right)}{P_{i} d \Omega_{r} \cos \theta_{r}}
$$

This expression basically describes the reflection as the measurement of the integrated, reflected power or flux, $P_{r}$, per unit projected solid angle, $\Omega_{i} \cos \theta_{r}$, in relation to the total incident power, $P_{i}$. The solid angle $\Omega_{r}$ can be associated with the dimension of the detecting element (i.e. pixel size) and defines the angular resolution. An integration of the BRDF over all angles $\theta_{r}$ and $\Phi_{r}$ represents the hemispherical reflectance, whose absolute value could be obtained with a proper calibration or a separate hemispherical measurement.

The acceptance angle, $\varphi$, creates a cone with the opening angle defined by $\varphi$. With the coordinate system centered at the specular peak (see box in Figure 1), this can be associated with the corresponding elevation or opening angle, $\theta_{d}$, that forms a circle for all pairs of $\left\{\theta_{d}, \Phi_{d}\right\}$ with $\Phi_{d}=0-2 \pi$. The specular reflectance at any given $\varphi$ is then calculated with equation (3), where the BRDF integrated up to $\varphi$ is divided by the total integrated BRDF and then multiplied by the hemispherical reflectance value $\rho_{h}(2 \pi)$. If $\rho_{h}(2 \pi)$ is not known the function is simply normalized to 1 .

$$
\rho_{s}(\varphi)=\int_{\theta_{d}=0}^{\varphi} \int_{\Phi_{d}=0}^{2 \pi} B R D F\left(\theta_{d}, \Phi_{d}\right) / \int_{\theta_{d}=-\frac{\pi}{2}}^{\frac{\pi}{2}} \int_{\Phi_{d}=0}^{2 \pi} B R D F\left(\theta_{d}, \Phi_{d}\right) \rho_{h}(2 \pi)
$$

The reflectance as a function of acceptance angle ROA is a plot of $\rho_{s}(\varphi)$ per $\varphi$, where a collector designer for example can read the value of interest for his application. It can also be of interest to plot the function as 1- $\rho_{s}(\varphi)$ to represent the losses from the hemispherical reflectance in relation to $\varphi$. In practice, a triangulation algorithm needs to be performed on the data first to find the integrated volume of the BRDF, because the measured data set consists only of discrete points with a given resolution. We apply a Delaunay triangulation algorithm.

\subsection{Prototype design}

This parallel catadioptric imaging goniophotometer [11] features as main component a mirrored ellipsoid (catoptric part). The two dimensional counterpart of an ellipsoid is the ellipse, which is defined by its two focal points F1 and F2. The focal points are located on one axis defined by the mayor half axis, $a$. The minor half axis, $b$, continues perpendicular to $a$. The ellipsoid is created by rotating the ellipse around the major half axis with a rotation angle $\pi$. Any light ray coming from one focal point is redirected by the ellipsoid walls towards the other focal point (Figure 2). If the incoming light bundle is composed of parallel light, the ellipsoid creates a junction point at half the distance between wall and F2. A camera with a $180^{\circ}$ fisheye lens (dioptric part) is positioned in the focal point $F 2$ and creates an image of the light distribution, where each pixel represents one scatter angle $\left\{\theta_{d}, \Phi_{d}\right\}$ in which the light left the sample. The sample holder uses precision screws to position the sample, which can be a first or second surface mirror, in the focal point $F 1$.

Since the BRDF of typical solar mirror materials is composed of a high intensity specular peak and a low intensity scatter, it is necessary to mask the specular peak when measuring the scatter signal with increased exposure time. For this purpose the instrument features two perpendicular shadow strips that rotate along the ellipsoid wall and are mounted with their rotation axes along the minor and mayor axes of the ellipsoid (Figure 2). For measurements of the scatter signal, first one, then the other shadow strip is positioned manually to cover the specular peak. Each strip also masks part of the scatter signal along its axis, which is why two shadow strips are necessary. The complete BRDF measurement of a mirror sample is a composite of peak measurement and several shadow measurements with the peak partly and completely covered. Without the shadow strips it would be impossible to distinguish the near specular scatter from the peak. 
A great part of the instrument design depends on the availability of adequate off the shelf camera and lens components. A beneficiary trade-off between their properties and the ideally required properties must be found. The theoretical angular resolution depends on the combination of sensor array and lens field of view. We decided on a $180^{\circ}$ Sigma circular fisheye lens with $f=4.5 \mathrm{~mm}$ focal length. This lens produces a circular image on the $4 / 3$ CCD detector array with 3296 x 2472 pixel. Therefore an angular resolution of around 1.3 mrad can be expected if the lens produces linear mapping over the whole hemisphere. The selected CCD array features a fill factor of $80 \%$ to minimize losses due to nonsensitive areas between pixels.

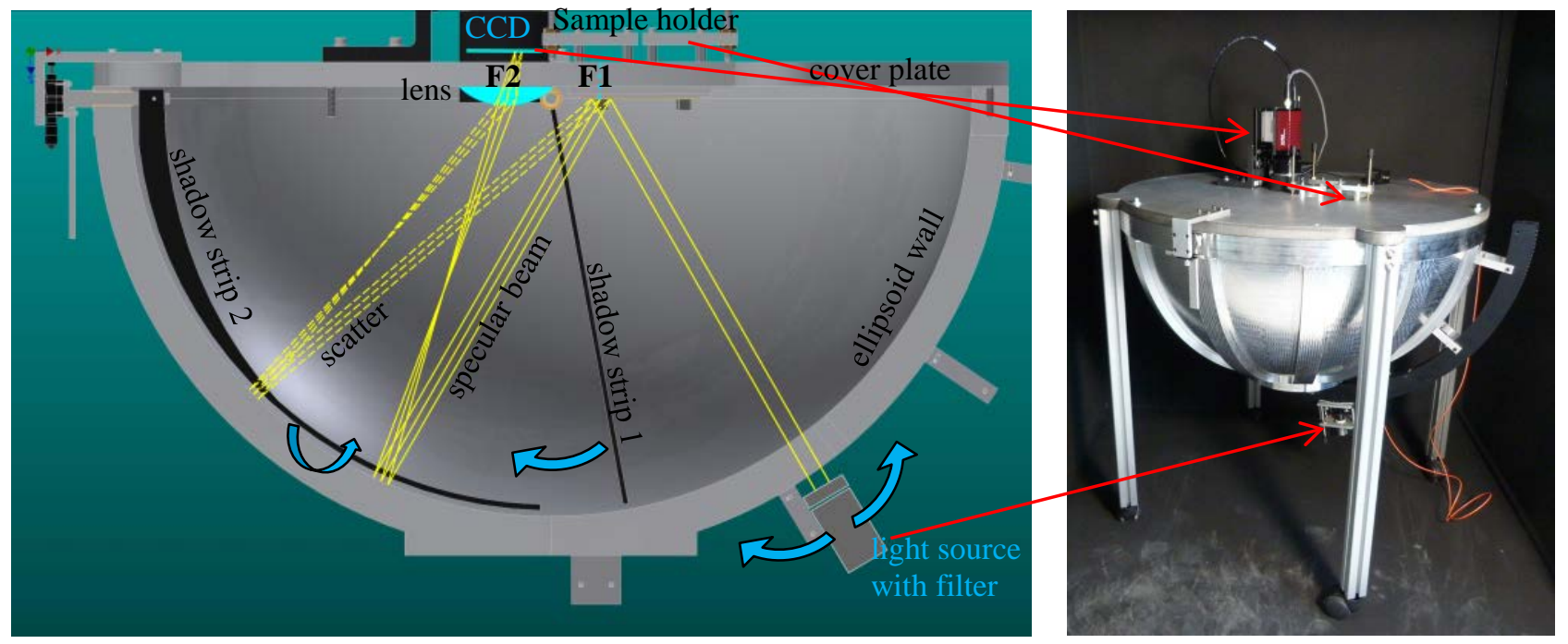

Figure 2: Working principle of the MIRA prototype that can be seen on the right. The light source illuminates the sample with parallel, monochromatic radiation at the first focal point $F 1$ of the ellipsoid. The mirrored ellipsoid wall redirects all radiation that comes from the sample towards the second focal point $F 2$, where a camera with a $180^{\circ}$ fisheye lens is situated. Each pixel position in the image represents one direction of the reflected light coming from the sample. To distinguish the low intensity scatter (dotted lines) from the high intensity specular peak of the reflection (continuous lines), the peak can be masked with a shadow strip.

In order to capture the BRDF, the lens if focused on the latent image that is created by the ellipsoid wall at half the distance between wall and lens. This latent image of the BRDF has roughly an ellipsoidal shape. The lens is focused at the distance $R$, where $R$ can be assumed to be $R \approx b / 2$, if the eccentricity of the ellipsoid is small (eccentricity $e=$ $\sqrt{b^{2}-a^{2}}$ ). $R$ must not be smaller than the minimal object distance of the lens, which is a first design parameter for the size of the ellipsoid ( $b>$ minimal object distance times two). The eccentricity of the ellipsoid determines the variance of $R$ in the 3-dimensional space. This must be within the depth of focus of the lens for the greatest numerical aperture. Interestingly, in contrast to our design, existing designs of other parallel catadiopic imaging goniophotometers mostly feature a very big eccentricity [7] [12][13].

Raytracing showed that distortion aberrations are minimal for an ellipsoid with $a \equiv b$ (thus a perfect sphere) and become larger as $|b / a-1|$ increases [14]. Using a sphere is impractical since sample and detector would have to be positioned at the same focal point. The relative dimensions of the ellipsoid, especially its eccentricity are chosen to be as small as possible considering realistic dimensions of sample and optical imaging system to avoid them disturbing each other. Since $e$ and $b$ are limited by practical considerations, $a$ can be derived from its relationship to the eccentricity. Raytracing also showed that aberrations increase for light rays that originate at increasing distances from the focal point F2. This means that the measurement spot should be kept small, while still being large enough to cover a sufficient portion of sample surface roughness. After these considerations, our prototype features an ellipsoid with roughly $690 \mathrm{~mm}$ diameter and $345 \mathrm{~mm}$ height and an eccentricity of $e=37 \mathrm{~mm}$. The measurement spot has a diameter of $3.5 \mathrm{~mm}$.

This is a reasonably compact design but reaches the limit of the technically feasible when it comes to producing the actual ellipsoid with sufficient precision. The system is very sensitive to divergence of the ideal beam path due to the entrance pupil of the optical imaging system. This is expressively explained in [12][15]. Diversion comes mainly from shape errors of the ellipsoid and a displacement at $F 1$. The production requirement for the ellipsoid shape precision is defined by the maximal acceptable deviation of the surface normal from the ideal shape at each point on the ellipsoid 
surface, $\beta$, which in our case was $|\beta|<0.5 \mathrm{mrad}$. The ellipsoid was fabricated from a massive aluminum block, milled to an approximate shape at Hatec Plattling $\mathrm{GmbH}$ (Germany) and in a second step was polished to its final shape and finish with a precision diamond milling machine at Kugler GmbH (Germany). The shape was checked and accepted at the fabrication site with a tactile instrument measuring at 200 uniformly distributed points. Later we found out that this was not an adequate surface inspection, as the 200 measurement points were uniformly distributed in a 2-dimensional plane but when projected into the ellipsoid surface, congregated closely at the steep edges of the walls and were widely spaced at the center area. Thus, the measurement result was misleading because of missing local information. The actual shape quality is discussed in section 4.2 .

\subsection{Angular mapping}

Due to the angular to special mapping function of the system, each illuminated pixel on the detector array represents one angle $\left\{\theta_{d}, \Phi_{d}\right\}$, in which a light-ray left the sample. All parallel rays that left different locations on the sample in the same direction (within the boundaries discussed in section 4.1) congregate on this one corresponding pixel. If only few rays are scattered in a specific direction, the intensity and thus the grey value at that pixel location will be low. The image is therefore a direct representation of the BRDF, averaged over the illuminated surface area. Depending on the incidence angle and therefore the angle of the specular direction after reflection on the sample, $\theta_{d \text { Peak }}=\theta_{r}$, the specular peak of the intensity distribution is located at its corresponding position on the detector array and not at its center. Additionally, theoretical discussions on other parallel catadiopic imaging goniophotometer concepts [7][12][13] position the imaging optics perpendicular to the plane that the foci F1 and F2 lay in. This requires a complex algorithm to calculate from the illuminated pixel position on the detector array the angle in which a light ray left the sample.

When only near specular scatter in relation to the specular peak is of interest, we can do without a complex algorithm by centering the polar coordinate system in the image at the specular peak $\theta_{d \text { Peak }}=0$. The calculation with equation (3) is then performed by calculating all other offset angles, $\theta_{d}$, by relating the corresponding pixel distance to the specular peak position with the angular resolution as seen in Figure 3.

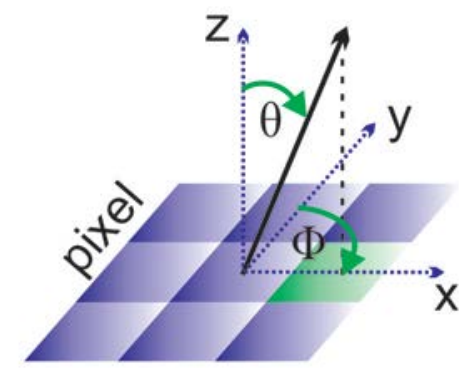

Figure 3: The conversion of the pixel positions for each BRDF data point into polar coordinates is performed with the polar coordinate system centered at the specular peak position. The offset angle from specular peak, $\theta_{d}$, is calculated by relating the pixel distance to the specular peak with the angular resolution of the instrument.

This method requires a constant angular resolution over the whole field of view. This was tested using a diffraction grating placed at the sample position and illuminated with varying wavelength bands and incidence angles. The angle between the first and zero order at the illumination wavelength corresponds to $\theta_{d}$, if the zero order position is considered as the specular peak position. It can be calculated using the common grating equation and gives the angular resolution in this local area. The test revealed a constant angular resolution of 1.3 mrad per pixel with a standard deviation of 0.05 mrad. No distortion coming from the fisheye lens was detected. 


\section{EXPERIMENTAL}

The availability of scanning goniophotometers that are able to measure the near specular scatter of highly specular materials with the necessary angular resolution of $\sim 1 \mathrm{mrad}$ or better and the required dynamic range is scarce. We found this at Pab Advanced Technologies Inc. (in the following "PAB") in Germany, where we sent exemplary solar mirror material samples for reference measurements. The next sections briefly explain the MIRA validation approach, present the comparison measurements and discuss the results. In the last section of this chapter we also present other useful applications of this measurement approach.

\subsection{Validation approach}

Two typical CSP glass-alternative reflector materials were sent to PAB for reference measurements: silvered polymer film material and enhanced aluminum sheet with a protective top coat. These measurements were performed in 2011, at the time the instrument was being designed. Due to huge delays in fabrication and assembly of the prototype the actual measurements with MIRA could only be done in 2014. A change of the reflective properties of the samples can therefore not completely be excluded.

PAB provided BRDF data only, measured up to an angle $\theta_{d}=95 \mathrm{mrad}$ at $\lambda=633 \mathrm{~nm}$. We performed the calculation of the reflectance as a function of acceptance angle (ROA) according to equation (3) with the same algorithm on both datasets (PAB and MIRA). Since the integration algorithm assumes the complete integrated BRDF to be equal with the total reflected signal, i.e. the hemispherical reflectance, we used $\theta_{d}=95 \mathrm{mrad}$ as the cutoff angle in the MIRA integration for comparison reasons. The total integrated volume at $95 \mathrm{mrad}$ was used here for the normalized ROA function. MIRA measurements were performed on 4 locations on the sample, PAB measurements only on one location. The materials were selected because we typically measure a difference of several percentage points between hemispherical reflectance and specular reflectance obtained with an off the shelf reflectometer (Devices and Services 15R [3] in the following called "D\&S") at $\varphi=12.5 \mathrm{mrad}$ and $\lambda=660 \mathrm{~nm}$. The samples were measured with MIRA at $\lambda=633 \mathrm{~nm}$, which is equal to the measurement wavelength as in the $\mathrm{PAB}$ measurement.

\subsection{Measurements}

Measurements have been performed on two typical glass alternative solar reflector materials: Silvered polymer film and an enhanced, aluminum sheet material with a protective top coat. The polymer film is very flexible and must be applied to a substrate. It can reach very high hemispherical reflectance values that compete with solar thin-glass mirrors. The material itself however displays a surface structure that is visible by the eye and its specular reflectance is also influenced by the smoothness or roughness of any substrate it is applied to. The aluminum material is composed of a rolled aluminum sheet substrate of about $1 \mathrm{~mm}$ thickness with layers of pure aluminum, reflective enhancing coatings applied by physical vapor deposition (PVD) and a protective top coat. The exact layer composition varies depending on product and producer. The protective layer has a thickness of approximately 3 microns and produces a typical interference pattern in the reflectance spectrum, which can shift phase depending on local variations on the sample. These properties make it more difficult to compare measurement results and make the producers of these materials dependent on reliable measurement techniques, so that their product is not penalized on the market due to misleading measurement results. The rolling marks produce a directional pattern in the surface which is responsible for a gloss line perpendicular to the rolling pattern.

The BRDF surface plots obtained MIRA reproduce the shape of PAB measurements for both materials as can be seen in Figure 4 and Figure 5. However the peak of MIRA measurements appears wider due to the not corrected instrument signature. The gloss line of the aluminum BRDF is clearly visible, while the polymer film reveals an inhomogeneous scattering pattern. Both could influence the measurement result depending on the applied measurement system. 
BRDF from PAB

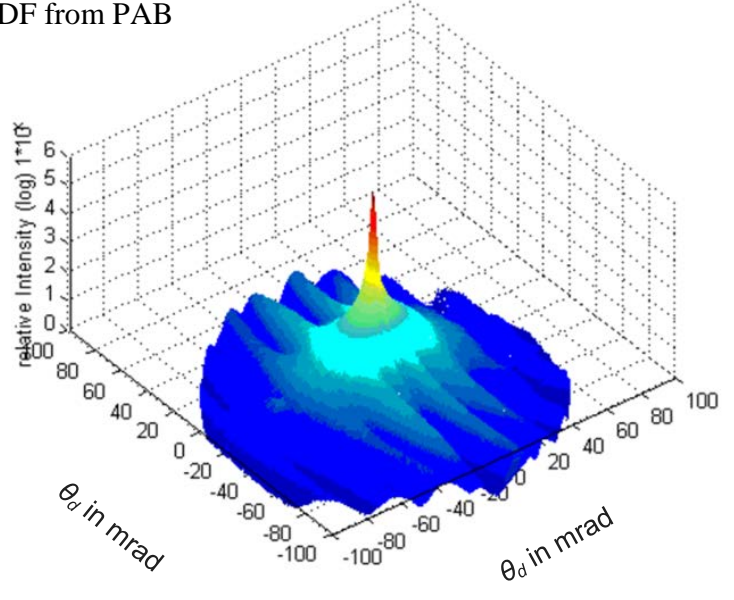

BRDF from MIRA

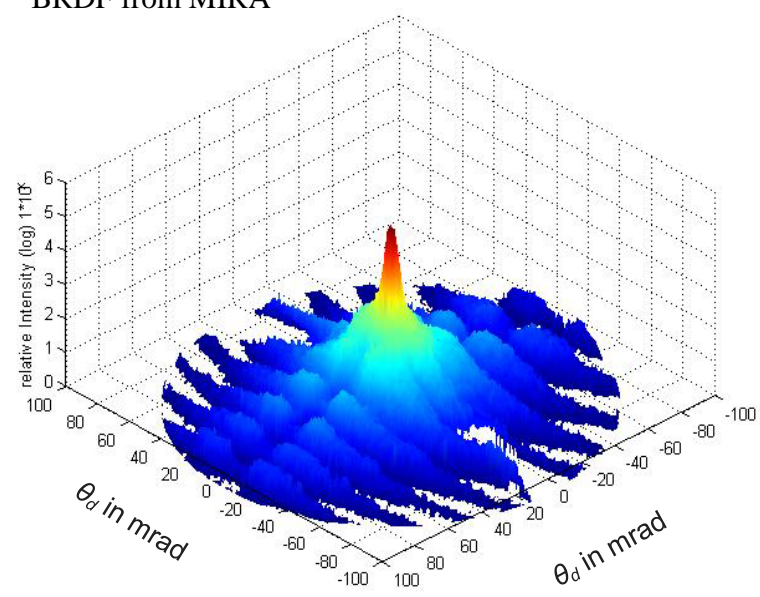

Figure 4: BRDF surface plots of the polymer film sample measured with MIRA and PAB. They show a comparable shape and reveal an inhomogeneous scatter pattern of the material. Both plots include the signature, which is wider for MIRA.

BRDF from PAB

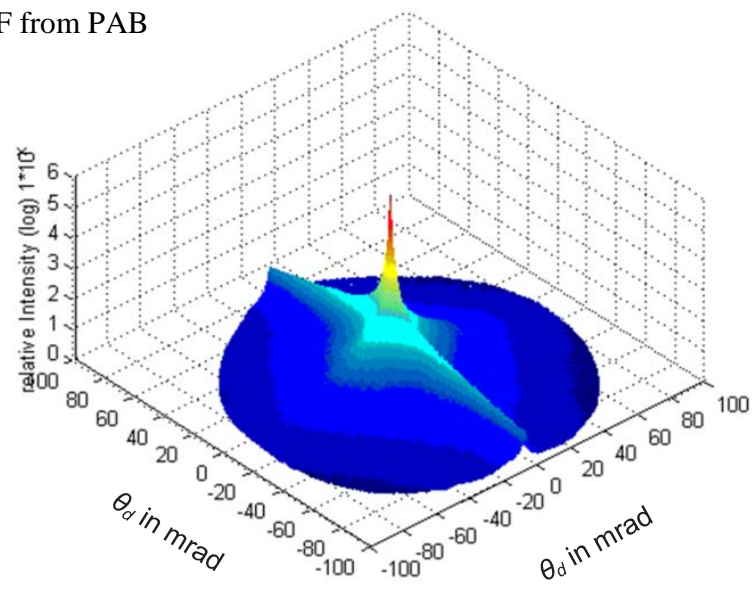

BRDF from MIRA

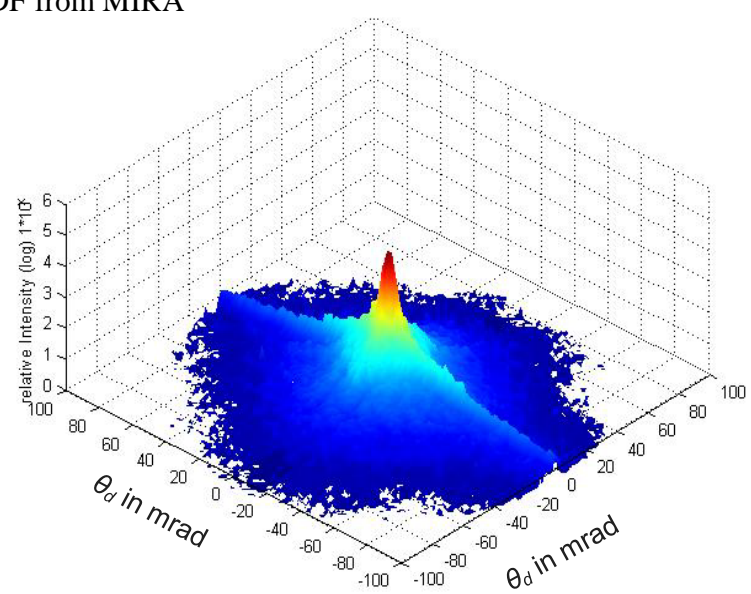

Figure 5: BRDF surface plots of aluminum sample from PAB and MIRA agree well and show the characteristic gloss line. Both plots include the system signature, which is wider for MIRA. The plots indicate that a small part of the scatter signal continues at larger angles than $\theta_{d}=100$ mrad. For comparison reasons with PAB, MIRA integration was performed up to a cutoff angle $\theta_{d}=95 \mathrm{mrad}$. 


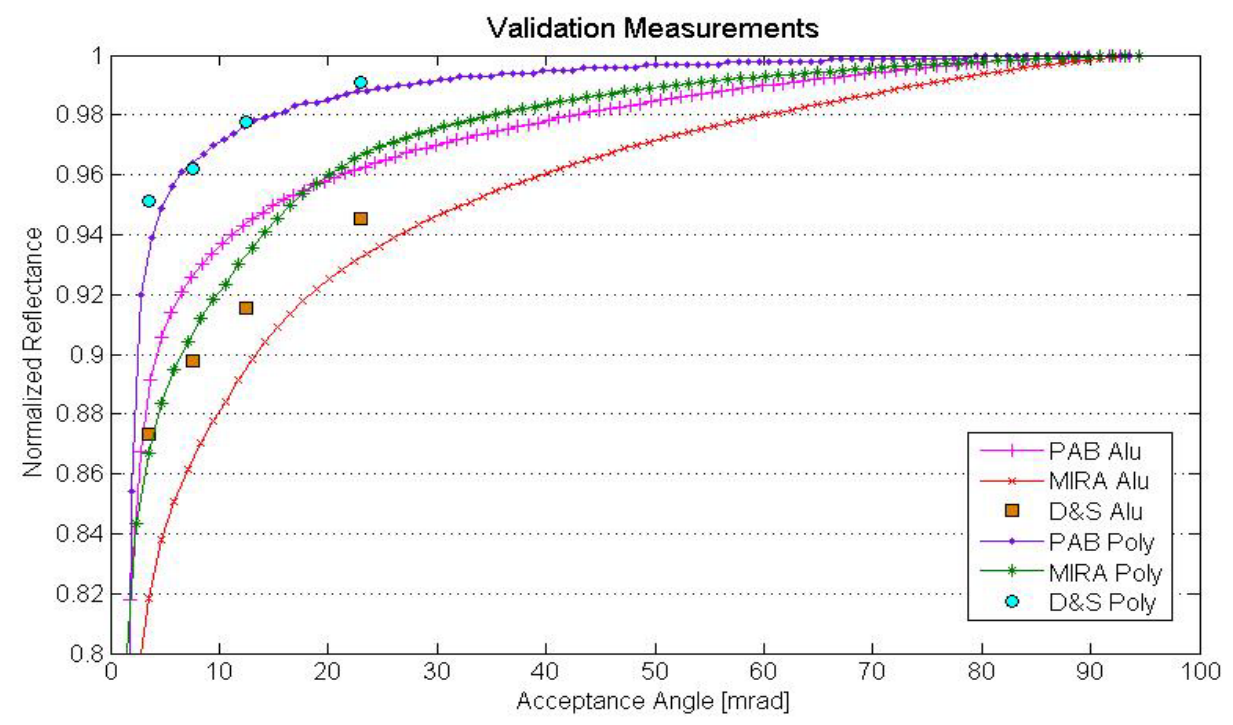

Figure 6: Plots of reflectance as a function of acceptance angle (ROA) of polymer film and aluminum samples that have been measured with MIRA and PAB. The results agree with a difference of $<5$ percentage points at $\varphi>10$ mrad. Aluminum sample might suffer from degradation but still the differences are considered to be mainly due to the instrument signature of MIRA.

The ROA functions presented in Figure 6 for both materials agree with a difference of $<5$ percentage points at $\varphi>10$ mrad. D\&S measurements agree almost exactly with PAB results for the polymer film sample, which indicates, that those represent the real value better than MIRA.

The plotted ROA results of MIRA represent the average of 4 measurements. The aluminum measurements vary greatly depending on the measurement location on the sample and D\&S measurements are also lower than PAB results by $\sim 2$ percentage points. This might indicate a degradation of the sample during the 3 years that lie between measurements. Scratches on the surface were observed at the time of measurement.

The aluminum sample was used to test the reproducibility of the instrument. Ten measurements were performed over two days without changing the sample position. The results show a maximal difference in the ROA curve of less than 0.3 percentage points with an average of 0.08 percentage points.

\subsection{Discussion}

The BRDF surface plots measured on the two reflector materials by MIRA and PAB show a comparable shape although the BRDF obtained with MIRA shows a wider peak due to the large instrument signature (see section 4.3). The ROA plots from MIRA agree with PAB data with a difference $<5$ percentage points at $\varphi>10$ mrad. The agreement becomes less accurate at acceptance angles $\varphi<10 \mathrm{mrad}$. It is very probable that this is in part due to the pronounced instrument signature of MIRA.

Comparing specular reflectance results obtained by two different measurement systems depends on many factors such as measurement wavelength, spot size, equal acceptance angle for center and border areas of measurement spot, incidence beam diversion, instrument signature, instrument uncertainty, local sample homogeneity and changes of sample properties between measurements. Since 3 years lie between PAB and MIRA measurements, the latter could also be in part responsible for the differences. PAB as well as D\&S instruments have a spot size of $10 \mathrm{~mm}$ diameter, while MIRA spot size is of $3.5 \mathrm{~mm}$ diameter. Both spot sizes are sufficient to capture surface structures responsible for light scatter in the visible. But the local variations on the aluminum sample have a higher impact on MIRA results, since the bigger spot size of the others averages local variations within the measurement spot.

The measurement uncertainty of PAB measurements in this case was not specified but we assume it to be less than the observed difference of the results. For CSP applications a measurement uncertainty around \pm 1 percentage point is desirable. These first measurements show that the MIRA concept and data treatment serve its purpose well, although a higher accuracy would be preferred. This could be established by taking the measures discussed in the next section. 
Another set of comparison measurements with new reflector samples was being performed by the time this paper was finished but results were not available yet. An improved version of the instrument prototype promises a better accuracy at narrow acceptance angles.

\subsection{Application possibilities}

Research on aging or soiling of the reflector materials is another field of application where the MIRA concept shows advantages over the conventional specular reflectance measurement methods. Their measurement accuracy decreases with less specularity of the mirror sample, which happens when degradation or soiling of the surface destroys the specular peak and increases the scatter part. BRDF analysis accuracy is not influenced by this. It can give hints on the way the degradation affects the reflectance by evaluating the BRDF shape and by plotting the ROA losses (see Figure 7). In the example in Figure 8, the sample has been exposed for one year at an unprotected, windy desert site. The constant impact of sand particles carried by the wind has had a kind of Gaussian effect on the sample that smoothed out the directional sinus surface pattern. The scatter thus has become stronger but less directional.

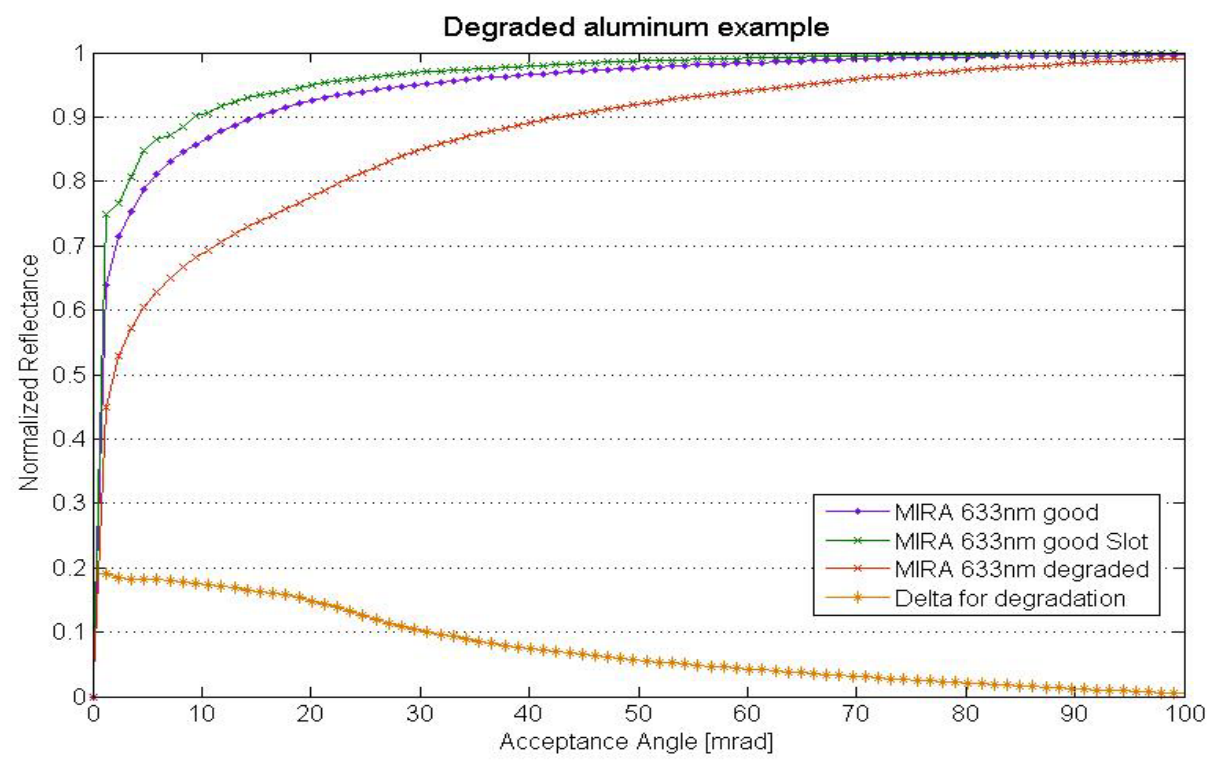

Figure 7: Measurement example of an aluminum mirror that has been exposed for one year at aggressive conditions in an unprotected, windy desert environment. The influence of a change in surface properties on specular reflectance can be demonstrated with the ROA function (compare green dotted line to blue continuous line or the plotted losses in the yellow line). The green (-x-) line shows the ROA result of the integration for the "good" measurement using a slot aperture that opens parallel to the direction of the gloss line. This represents a more adequate evaluation if this kind of material is meant to be used in a line-focusing collector. 

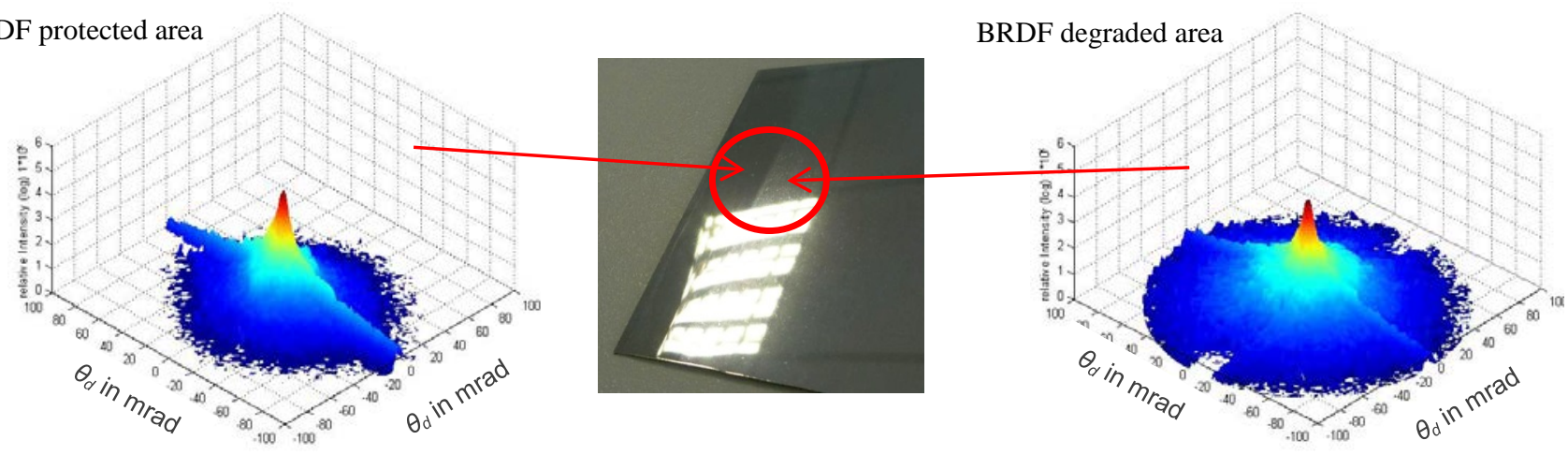

Figure 8: BRDF surface plots of the aluminum samples that has been exposed for one year at aggressive conditions in an unprotected, windy desert environment. The exposed area shows visible abrasion (surface plot right), while the area that was protected by the clamp of the mounting structure is less affected (surface plot left).

The measurement of the BRDF of mirror materials is useful to characterize their near specular scatter behavior. It also gives hints on bidirectional behavior of the reflected light, as is common with rolled aluminum mirrors. For those materials, it is not always adequate to use a circular acceptance aperture for measurements as is the case for a conventional specular reflectometer. The obtained result misinterprets the real performance this particular mirror material could have in a line-focusing collector system, where the scatter in the direction parallel to the focus line does not lead to energy losses. When the BRDF is known, a virtual slot aperture can solve this problem and evaluate the mirror material correctly in accordance with the application it is meant for. As can be seen in Figure 7, these different evaluation approaches lead to differences in results between 3-5 percentage points. The slot integration favors this particular material much more for line-focusing collector systems, as is the commercially most common parabolic trough CSP collector.

\section{LESSONS LEARNED FOR IMPROVEMENTS}

The concept of the parallel catadiopic imaging goniophotometer that uses a mirrored ellipsoid and some kind of wide angle imaging system is appealing but at the same time imposes a challenge to actually build a working system. None or only few systems we know of have been built and demonstrated their functionality. Limitations are inflicted by unchangeable properties of the components and the theory behind the concept itself. This is theoretically discussed in [15][16][17][18]. Other limitations presented here are part of this specific instrument prototype and can be overcome in an improved version. The following sections concentrate on the view angle, the shape quality of the mirrored ellipsoid and the system signature.

\subsection{View angle}

At first glance, the most appealing property of a parallel imaging goniophotometer is the seeming possibility of capturing the whole hemisphere above a sample in one single image with the help of a $2 \Theta=180^{\circ}$ (full view angle) fisheye lens. As Karamata et al. shows in [15] this fails in practice due to the position of the entrance pupil, which changes for rays incident at the lens surface at flat angels, nearing the edge of its field of view. Our experiments with MIRA confirmed his theoretical analysis. Karamata assumes a point of view from the lens which would "see" different areas at the sample each time more distant from the focal point F1, when approaching the edge of its field of view. In turn, a limited area of illumination at the focal point on the sample as is the case here (illuminated spot diameter is $3.5 \mathrm{~mm}$ ), leads to blindness of the lens when scattered light exceeds a limiting view angle $\Theta_{\text {lim }}$. A simple experiment that is independent of a correct incident light alignment can demonstrates this. With a highly specular mirror placed at $F 1$, the reflection of straylight entering through the incidence slot in the ellipsoid can be seen with the camera at the same time as the actual illuminated entrance slot (see Figure 9 right). Using simple geometry, $\Theta_{\text {lim }}$ can be calculated from the position of markers in the incidence slot at the edge of visibility. The flatfield image that is illuminated by a lambert source in Figure 9 (left) confirms this calculation after angle to pixel mapping according to the measurement resolution. For the MIRA setup, we found $\Theta_{\text {lim }} \sim 64^{\circ}$, which means that about $28 \%$ of the fisheye field of view is not exploited. Experiments of changing the lateral position of the camera for accessing angles $>\Theta_{\text {lim }}$ did not result effective. Increasing the measurement spot would increase the field of view, but would also introduce more aberrations for rays farther away from the focal points. 
An exemplary super wide angle lens with $2 \Theta=116^{\circ}$ view angle and $f=8.5 \mathrm{~mm}$ focal length would lead to a bigger entrance pupil and a higher resolution of 0.8 mrad per pixel.

The limited view angle has two consequences. First, the instrument's application is limited to the measurement of materials that do not display far angle scatter outside of $\Theta_{\text {lim. }}$. Second, when measuring at incidence angles much greater than near normal, $\theta_{i}$ of the measurement must be smaller than $\Theta_{\text {lim }}+\theta_{d, \max }$. Thus the application is also limited to measurements at incidence angles well below $\Theta_{\text {lim }}$. Actual solar reflector materials do show near specular scatter, but from our experience this stays at $\theta_{d, \max }<20^{\circ}$. The MIRA prototype thus is perfectly capable of analyzing their scatter behavior up to incidence angles of $\theta_{i}=45^{\circ}$.

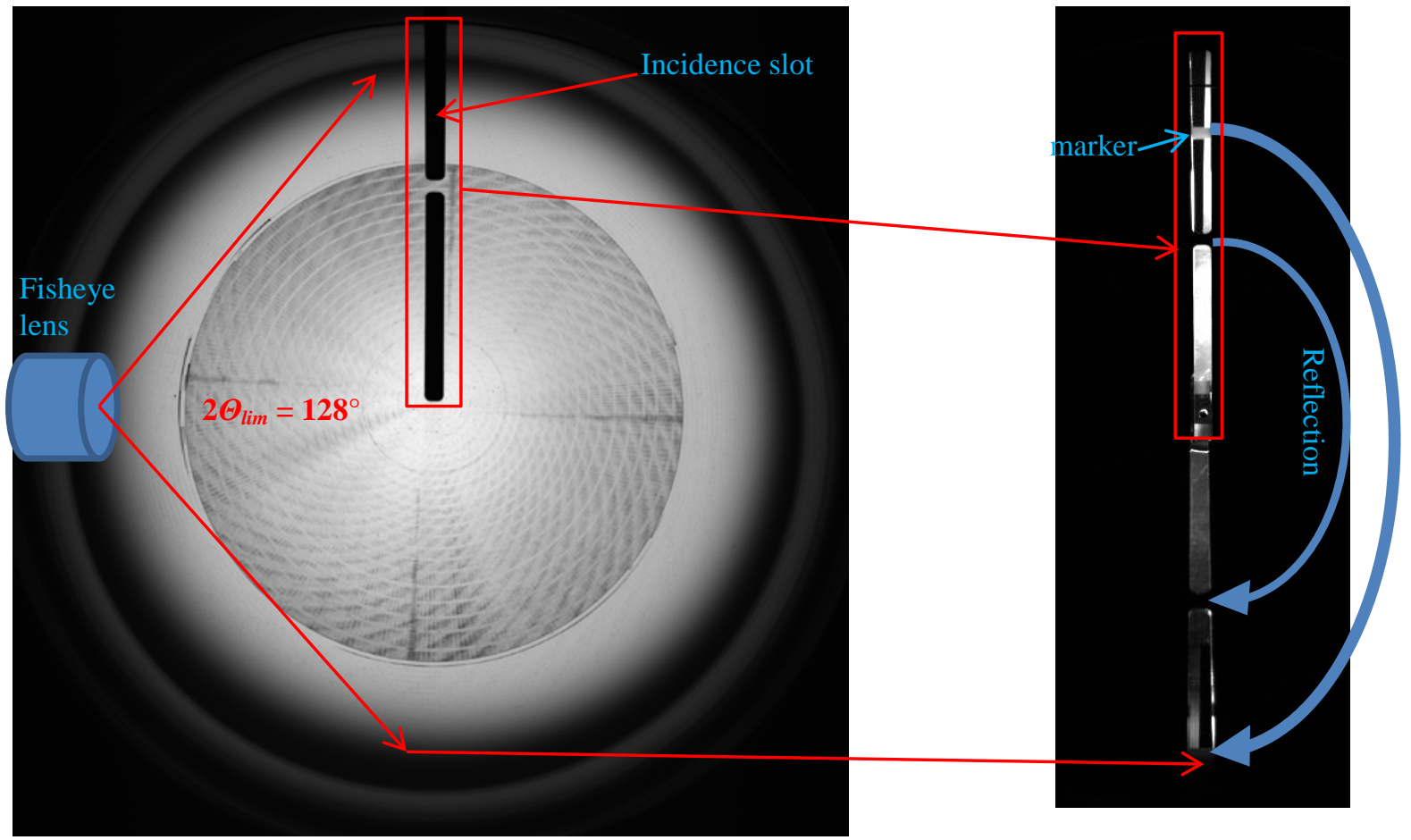

Figure 9: Left: The measurement image of a lambert source at $F 1$, which would result in a homogeneously illuminated image in case of a perfect ellipsoid and object lens. Here however, the dark areas clearly show markings from the production process, where the ellipsoid shape is imperfect and light misses the entrance pupil. Right: The image of straylight that enters through the incidence slot and is reflected by the ellipsoid towards the lens. By the position of markers in the incidence slot and the position of their reflection the maximum visible incidence angle can be detected. Both images serve as a confirmation for the real, limited view angle $\Theta_{\lim }$ of the fisheye lens in this setup.

\subsection{Ellipsoid imperfections}

Although the acceptance test which checked the ellipsoid fabrication quality with the tactile measurement head was approved, we discovered irregularities of the ellipsoid shape later during the validation process. As can be observed in Figure 9 (left), a large area around the center of the ellipsoid is covered with a circular pattern that was created by the milling head. The pattern is revealed when a lambert source is placed at the sample position in $F 1$. A uniformly illuminated area filling the whole field of view only influenced by possible aberrations of the lens would be expected for this measurement. The pattern however is clearly an ellipsoid surface property and extends up to a view angle of $\Theta_{\text {cam }} \sim$ $43^{\circ}$ seen from the camera and $\Theta_{\text {sam }} \sim 35^{\circ}$ seen from the sample. This means that all measurements with incidence angles $\theta_{i}<35^{\circ}$ are influenced by this pattern. It demonstrates the theory that rays that are reflected from the ellipsoid wall at local points with a too high slope deviation miss the entrance pupil and therefore the image becomes darker at the corresponding pixel position. This alters the measured BRDF and must be corrected.

Any optical system owns a unique fingerprint that modulates the signal that goes through it and also introduces systematical aberrations. An object with uniform luminance usually does not result in an image with equally uniform intensities. In astronomical observation it is common to take a so called "flatfield" image of the uniform blue sky for 
correction of this effect. We do the same by using the image in Figure 9 as our flatfield image. The correction algorithm creates a matrix of correction factors for every pixel, so that they are elevated or suppressed to an average grey value. This matrix not only corrects the surface irregularities, but also an intensity gradient that comes as vignetting from the lens.

Although this correction improves the measurement reliability, the BRDF information that misses the entrance pupil cannot be restored. This is especially of disadvantage if the BRDF is very irregular and introduces a higher measurement uncertainty. The exemplary measurements presented in section 3.2 show however, that the so corrected results are still reasonable. An ellipsoid created with a different fabrication approach or dimensions that are easier to manage with a milling machine could improve the measurement accuracy.

\subsection{Instrument signature}

The system signature is present in any BRDF measurement and represents the result obtained from a measurement without sample, directly of the illumination beam. Beam divergence, aberrations of the imaging optics, stray light and any other systematical errors produce a signature. Literature offers little solutions for a proper data treatment. The best advice is to reduce the instrument signature to a minimum [19]. A prominent signature makes it harder to resolve near specular scatter.

The signature of the actual MIRA prototype acts as a mayor source for differences in measurement results when compared with others. With the actual collimation divergence of $3.3 \mathrm{mrad}$ it can be calculated that the light source would illuminate an area of 3 pixel diameter at the CCD when free of scattering. However, measurements using a perfectly specular, first surface reference mirror reveal an illuminated area of 6-8 pixel diameter at the CCD. The most significant source for this comes from the object lens, since the ellipsoid shape imperfections are mainly responsible for "dark areas", but not for a widening of the spot. The signature does not change for measurements at different incidence angles, where different parts of the ellipsoid wall act in the redirection process. As can be seen in Figure 10, the MTF (modulation transfer function) of a sharp black to white edge taken with the camera-fisheye-system, shows a similar profile as the signature measurement. This confirms the assumption that the main problem comes from the lens.

Our actual treatment of the instrument signature for the calculation of the ROA function consists in the integration of the peak intensity inside the area dominated by the signature for the total integrated signal, but treating only the pixel outside of that as scattered light relevant for the ROA plot. A second approach using fitting functions is being investigated.

Using a lens with better MTF properties, which is usually not a fisheye, could improve the signature. Also a lens with a smaller field of view would improve the resolution and therefore the signature would impact less severe. The exemplary super wide angle lens with $2 \Theta=118^{\circ}$ view angle as mentioned in section 4.1 can improve the angular resolution to 0.8 mrad.
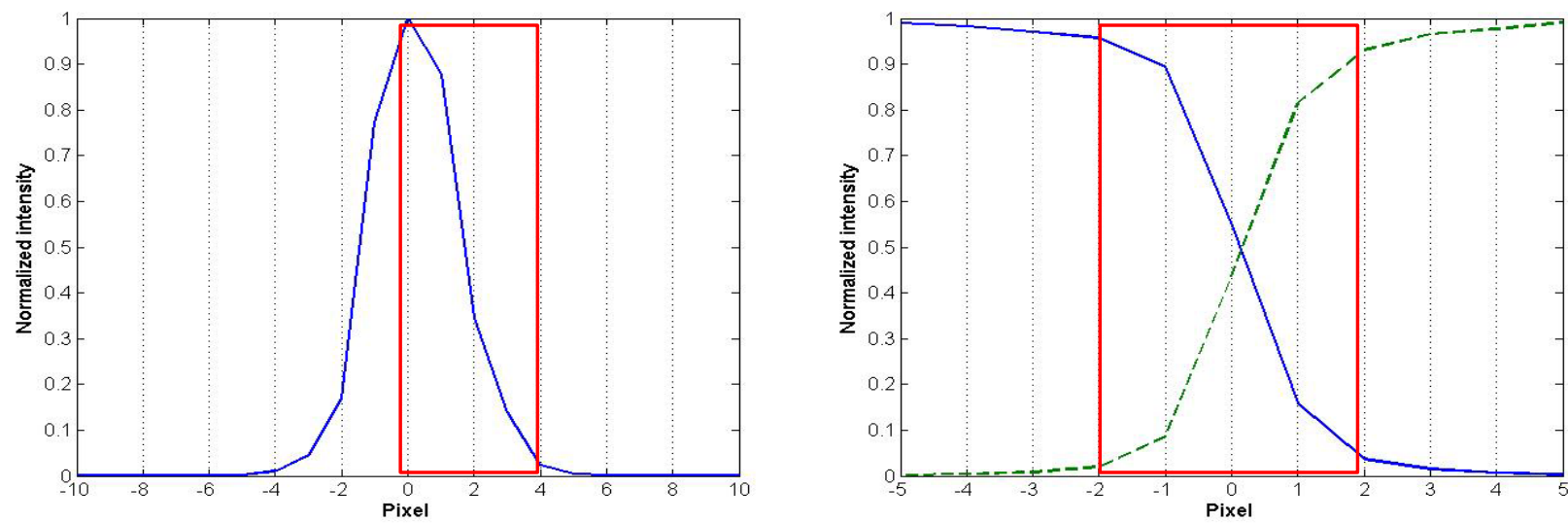

Figure 10: Left: Profile of the MIRA instrument signature using a perfectly specular reference mirror. With a resolution of 1.3 $\mathrm{mrad} / \mathrm{pixel}$ this corresponds to a radius of about $5 \mathrm{mrad}$. Near specular scatter can only be resolved outside of this range. Right: MTF of the object lens measured with a black to white edge (broken line) and white to black edge (continuous line). The slope of the signature profile is very similar to that of the lens MTF which indicates that the lens is a major contributor to the overall instrument signature. 


\section{CONCLUSIONS}

Due to the lack of proper off the shelf instrumentation for the analysis of specular reflectance including angular resolved near specular scatter, we developed a new instrument prototype based on the concept of a parallel catadioptric imaging goniophotometer at the OPAC laboratory in Spain. The purpose was to improve the available technology for evaluating glass alternative solar reflector materials, which might be disadvantaged with state of the art measurements due to their near specular scatter. The instrument employs a mirrored ellipsoid which redirects the light coming from the sample at one focal point, towards an optical imaging system using a fisheye lens at the second focal point. The measured BRDF of the sample can be used to calculate the specular reflectance as a function of acceptance angle (ROA) with an angular resolution of $\sim 1.3 \mathrm{mrad}$. Measurements at variable incidence angles up to $\theta_{i}=45^{\circ}$ and various wavelength bands in the visible range are possible.

Measurements of exemplary solar mirror samples were compared to measurements performed with a traditional scanning goniophotometer at pab Advanced Technologies Inc. The results agree with a difference $<5$ percentage points at $\varphi>10$ mrad, while increased differences were observed at narrower acceptance angles. A prominent instrument signature is mainly responsible for these deviations, although a correction is implemented in the data treatment. A detailed investigation of the systematical instrument limitations was conducted. Next to imperfections in the ellipsoid shape which can be corrected, the tests revealed the fisheye lens as the main source for the large signature. They also confirmed the theoretical expectation that the $180^{\circ}$ view angle of the fisheye lens cannot be fully exploited because of the shifting position of the entrance pupil. The actual view angle is therefore limited to $\Theta_{\lim } \sim 64^{\circ}$. A different lens could improve the overall performance of the instrument significantly. A super wide angle lens with a smaller view angle might serve to reduce the instrument signature, while additionally its bigger entrance pupil accommodates better for shape errors of the ellipsoid. The ellipsoid was designed to cover the whole hemisphere although the distribution of reflected light from solar mirror materials was expected to spread only about $\theta_{d}=10-20^{\circ}$. Using a whole hemi-ellipsoid or just part of it depends on the measureable incidence angles that are desired. A different ellipsoid size and design that eases the fabrication process could also improve the performance of the system. The presented suggestions can improve the performance of a follow up prototype towards the desired accuracy of \pm 1 percentage point.

The presented instrument design and method to analyze near specular scatter in form of a ROA function proves to be a new and well suited tool for evaluating solar mirrors and other specular materials. It can serve the concentrating solar industry well, when it comes to comparing the reflector material products or evaluating their influence on the overall performance in a CSP-collector system. It also serves well in durability and soiling research or any application where the near specular scatter analysis is essential. Due to the reduced field of view, the only limitation of the instruments application concerns materials that need the analysis of a full hemispherical BRDF.

\section{ACKNOWLEDGEMENTS}

The authors wish to acknowledge the effort of third parties that made the prototype assembly possible and thank the project executing organization BMU (German ministry for environment) for the financial support. They also thank Martin Hagmann, Alexander Oschepkov and Dr. Peter Apian-Bennewitz as well as the colleagues at OPAC for their contributions.

\section{REFERENCES}

[1] Stine, W.B., Harrigan, R.W., [Power from the sun], John Wiley and Sons. Inc. (1986).

[2] SolarPACES Reflectance Guideline "Parameters and method to evaluate the solar reflectance properties of reflector materials for concentrating solar power technology - Version 2.5" http://www.solarpaces.org/tasks/task-iii-solar-technology-and-advanced-applications/reflectance-measurementguideline (2013)

[3] Pettit, R.B., Freese, J. M., Mahoney, A.R., "The development of a portable specular reflectometer for monitoring solar mirror materials”, SPIE, Vol. 428 (1983)

[4] Heimsath, A., Kutscheidt, G., Nitz, P., "Detailed optical characterization of reflector materials for CSP applications“, Proc. SolarPACES 136 (2010) 
[5] Meyen, S., Fernández-Garía, A., Kennedy, C., Lüpfert, E., "Standardization of solar mirror reflectance measurements - Round Robin Test”, Proc. SolarPACES (2010)

[6] Ward, G.J., "Measuring and modeling anisotropic reflection” Siggraph '92 Chicago, July 26-31, Computer Graphics 26, 2 (1992)

[7] Karamata, B., Andersen, M., "Revisiting parallel catadioptric goniophotometers", Proc. SPIE Vol. 8788 87881Q-1 (2013)

[8] Meyen, S., Lüpfert, E., Pernpeintner, J., Fend, T., "Optical characterization of reflector material for concentrating solar power technology“, Proc. SolarPACES (2009)

[9] pab Advanced Technologies Ltd. web flyer, "Gonio-Photometer for reflection \& transmission measurements”, www.pab.edu.

[10] Apian-Bennewitz, P., “New scanning gonio-photometer for extended BRTF measurements”, Proc. SPIE 77920 (2010)

[11] German Patent DE 102012214 019.0, “Photogoniometrisches reflektometer - Mirror reflectance function analyzer (MIRA)” (2012)

[12] Rosete-Aguilar, M., Rodriguiez-Herrera, O.G., Bruce, N.C., “Optical design of a scatterometer with an ellipsoidal mirror”, Optical Engineering 426, pp. 1772-17777 (June 2003)

[13] Mattison, P., Dombrowski, M., Lorenz, J., "The hand-held directional reflectometer: An angular imaging device to measure BRDF and HDR in real-time”, Proc. SPIE 3426 (1998)

[14] Hagmann, M., "Analysis of the reflection properties.of solar mirror materials by measuring the BRDF to enhance the precision of state of the art methods” Diploma Thesis, Karlsruhe Institute of Technology (2012)

[15] Karamata, B., Andersen, M., “Origin and nature of measurement bias in catadioptric parallel goniophotometers" JOSAA, Vol 31(5), pp. 1040-1048, (2014).

[16] Gayeski, N., "New methods for measuring spectral, bi-directional transmission and reflection using digital cameras”, Master's thesis, Department of Architecture, Massachusetts Institute of Technology (2007)

[17] Andersen, M., Stokes, E., Gayeski, N., Browne, C., "Using digital imaging to assess spectral solar-optical properties of complex fenestration materials: A new approach in video-goniophotometry“, Solar Energy $84 \mathrm{pp}$. 549-562 (2010)

[18] Karamata, B., Andersen, M., "Limits and artefacts of reflective imaging goniophotometers for complex solar façade systems”, Proceedings EuroSun ISES Europe Solar Conference, Croatia (2012)

[19] Stover, J.C., Klicker, K.A., Cheever, D.R., Cady, F.M., "Reduction of instrument signature in near angle scatter measurements”, SPIE Vol. 749 Metrology: Figure and Finish pp. 46-53 (1987) 